\title{
Emotional Descriptors for Map-based Access to Music Libraries
}

\author{
Doris Baum, Andreas Rauber \\ Department of Software Technology and Interactive Systems \\ Vienna University of Technology \\ Vienna, Austria \\ http://www.ifs.tuwien.ac.at/mir
}

\begin{abstract}
Apart from genre- and artist-based organization, emotions are one of the most frequently used characteristics to describe and thus potentially organize music. Emotional descriptors may serve as additional labels to access and interact with music libraries. This paper reports on a user study evaluating a range of emotional descriptors from the PANAS-X schedule for their usefulness to describe pieces of music. It further investigates their potential as labels for SOM-based maps for music collections, analyzing the differences for labels agreed upon by a larger group of people versus strictly personalized labellings of maps due to different interpretations by individual users.
\end{abstract}

\section{Introduction}

The most prominent and dominant ways of organizing and describing music collections usually follow genre- or artist-based structures. Yet, music is also commonly described as carrying special emotions, evoking specific feelings. Quite frequently people listen to a wide range of musical styles, picking the specific type of music at a given situation according to their mood. Thus, automatically describing music according to emotional characteristics constitutes an interesting challenge to assist in interfacing with large electronic music repositories in diverse manners.

Yet, emotions are neither easily agreed upon, nor consistently assigned to music by different people. Research in emotions has a long and diverse history, and in spite of considerable efforts, no unanimous set of emotions to describe music has been agreed upon (a situation that emotions share with genres as class labels for music or even text documents). Worse, and again similar to the more conventional genre setting, emotions are not consistently assigned by different people to the same piece of music. Even more strongly than with musical genres, the attribution of certain emotional characteristics to a piece of music depend strongly on personal aspects, preferences, and - quite likely - on the emotional situation when the actual assignment task is being performed.

In spite of this highly volatile characteristic of emotions, they still seem to merit closer inspection to identify, in how far they may be used to support other, more conventional concepts in order to characterize music. We thus report 
on a user study which addresses three different aspects of emotions for music characterization, namely (1) in how far do people agree when assigning emotions to a given set of pieces of music; (2) in how far are the similarities between pieces of music assigned to the same emotional categories reflected in the respective feature representation, i.e. do the features we use to describe music for tasks like genre-based organization also support an emotional organization; and (3) while different people may assign different emotional attributes to a given set of music, may these personal labels still be used to describe an organization of music automatically using these emotional labels. Ten subjects took part in the study, labeling music from the benchmark collection created by George Tzanetakis according to emotions from the PANAS-X schedule [17. These were then analyzed with respect to inter-indexer consistency, as well as interpreted as labels mapped onto a self-organizing map (SOM) [6] trained on these pieces of music using the SOMeJB music digital library system [10122.

The remainder of this paper is structured as follows. Section 2 describes some related work in the fields of emotion analysis for music, and particularly its use in music information retrieval. The details of the user study performed are outlined in Section 3 followed by an analysis of the results in Section 4 . The main results as well as an outlook on ongoing work resulting from this study conclude the paper in Section 5

\section{Related Work}

Conventionally, music collections are structured according to artist- and genrestyle organizations, be it manually assigned such as in most current web shops or on-line portals, or automatically created via classification systems. The latter are mostly oriented towards western-style music, but there is increasing interest in similar organizations specifically targeted to traditional native music styles, such as e.g. for a Korean music digital library [7].

The relation between music and emotions has been addressed psychologically as early as 1936 by Hevner [5], who also created an "adjective circle" with 8 emotion categories. Since then, a lot of studies have been devoted to the emotional aspects of music, a substantial number of them summed up by Gabrielsson and Juslin 4. However, as Gabrielsson and Juslin conclude, there still is no universally accepted set of adjectives or other characterizations for classifying music according to emotion. Thayer proposed one of the psychological models for emotion: Thayer's Model of Mood [15. Therein, emotion is not defined as groups of adjectives but rather as a two-dimensional scale of Energy (calm - energetic) and Stress (happy - anxious).

The work of Tellegen, Watson, and Clark [14] led to the development of the PANAS-X schedule [17, which combines a dimensional scale of Positive and Negative Affect with adjective groups for a number of emotions.

Based on the vast pool of studies on emotional aspects of music, numerous groups have turned to different emotional models in the context of automated analysis of music. A set of emotional interpretations of certain parts of 
the Rhythm Pattern feature set is presented in [9]. The resulting Weather Charts were used to describe, i.e. label areas of the Islands of Music maps, clustering pieces of music according to perceived sound similarity. Apart from pure frequency spectra characteristics, such as low frequencies dominant, emotional aspects such as non-aggressiveness, based on the ratio of low-frequency amplitude modulations in the lower bark bands, were used to characterize certain areas on the map.

Li and Ogihara [8] use thirteen adjective groups - Farnsworth's [3] ten groups, which were in turn derived from Hevner's, plus three their test user created - to classify emotion in music with Support Vector Machines.

Yang and Lee [18] use the Positive and Negative Affect dimensions and the emotion categories of the PANAS-X schedule [17] and the Russel [13] and Tellegen-Watson-Clark [14] models. They extract 12 standard low-level features from the audio sources, generate 12 more features by genetic algorithms, and apply Support Vector Machine regression to them. Also, the lyrics of the songs are taken into account to disambiguate the emotion of the song.

Cunningham, Downie, and Bainbridge in [1] analyze the rather neglected area of dislike, even disgust, for music pieces. From 395 responses to a survey on "the worst song ever", they extracted, with a grounded theory approach, the most commonly named reasons why songs are "bad". Among these are the quality of the lyrics or voice, the "earworm effect", a dislike of the corresponding music video, perceived pretentiousness of the song, over-exposure to the music, and unpleasant personal experience associated with the piece.

\section{User Study}

\subsection{Music Collection}

The music used in this study was based on George Tzanetakis' benchmark collection [16. It consists of mp3s with 30 second extracts of songs from ten different genres: Blues, Classical Music, Country, Disco, Hiphop, Jazz, Metal, Pop, Reggae and Rock. From the full benchmark collection of 1000 songs, 20 from every genre were selected randomly to make up a collection of 200 songs. They were all named uniformly so as to not give any hints concerning the genre. The ordering of the songs was randomized when presenting them to the test subjects during a form-based evaluation session to further prevent any bias.

\subsection{Emotions}

As previously discussed, there have been a number of studies on music and emotions, but no standard set of emotions seems to have been established. Thus, a set of emotions had to be selected, which should be founded in psychology and would prove useful in the study. The emotional categories were taken from The PANAS-X Manual for the Positive and Negative Affect Schedule - Expanded Form by Clark and Watson [17. This may later allow us to bring together adjective groups and two-dimensional scales, as a Positive/Negative Affect axis 


\begin{tabular}{|l|c||l|c|}
\hline Category & \multicolumn{1}{|c|}{ Adjectives } & Category & Adjectives \\
\hline Fear: & scared / nervous & Attentiveness: & determined / alert \\
\hline Hostility: & angry / disgusted & Shyness: & timid / shy \\
\hline Guilt: & guilty / ashamed & Fatigue: & tired / sleepy \\
\hline Sadness: & sad / lonely & Serenity: & calm / relaxed \\
\hline Joviality: & happy / cheerful & Surprise: & surprised / astonished \\
\hline Self-Assurance: & proud / strong & don't know & other / unassignable \\
\hline
\end{tabular}

Table 1. Adjectives assigned to emotional categories, based on [17]

together with an Arousal / Activation level can form a two-dimensional model similar to Thayer's. The categories in the the PANAS-X Manual are: fear, hostility, guilt, sadness, joviality, self-assurance, attentiveness, shyness, fatigue, serenity and surprise. Each category was represented in the test questionnaires by two adjectives listed in Table 1 which the subjects could associate with the music.

\subsection{Study Set-up}

Ten subjects took part in the study, all between 20 and 40 years old, 6 male and 4 female, 9 of them with academic background (university students or graduates). Their musical expertise varies from amateurs to experts with theoretical background knowledge, playing instruments, singing, or even writing their own music. The subjects also showed a rather diverse range of preferred musical styles. Obviously, the small number of participants does not allow a purely quantitative evaluation, but requires a qualitative evaluation.

The study was conducted via a form-based on-line questionnaire. While data collection via the Internet form was basically open to the general public, most participants, and specifically the ones that the results in this paper are based upon, were selected to participate in the study. The home-page contained an explanation of the study for the subjects and provided a possibility to listen interactively to the individual pieces of music while ticking the check-boxes of the appropriate emotional characteristics. PHP scripts were used to automatically generate an individual music rating questionnaire for each test subject. A different randomized ordering of the songs was produced to prevent any potential bias introduced by the sequence of the pieces of music as well as the duration of the evaluation session.

The results of the study were analyzed in several different manners. First of all, the variation of emotions assigned to the various titles was analyzed to obtain a feeling for inter-indexer consistency, i.e. in how far the test subjects agreed with each other when assigning emotions to music.

Secondly, the pieces of music were clustered on a self-organizing map (SOM) $[6$ using Rhythm Pattern features [11. It groups the music according to sound similarity as expressed by the feature space on a two-dimensional map display in such a way that similar pieces of music are located next to each other. The resulting map was then labeled both with the respective genre labels as well as the emotional labels in order to see in how far consistent regions could be identified 


\begin{tabular}{|l|r|r|}
\hline EMOTION & $\mathbf{5 0 \%}$ & $\mathbf{7 0 \%}$ \\
\hline fear & 4 & 0 \\
\hline hostility & 22 & 7 \\
\hline guilt & 0 & 0 \\
\hline sadness & 25 & 3 \\
\hline joviality & 41 & 18 \\
\hline self-assurance & 36 & 19 \\
\hline attentiveness & 9 & 3 \\
\hline shyness & 2 & 0 \\
\hline fatigue & 9 & 3 \\
\hline serenity & 45 & 19 \\
\hline surprise & 0 & 0 \\
\hline
\end{tabular}

Table 2. Number of songs in the emotion categories, regarding all users, with $50 \%$ and $70 \%$ agreement required

in both visualizations, which do not necessarily have to coincide. The PlaySOM software 2] was used for the subsequent evaluations and visualizations.

\section{Results}

\subsection{Variations in emotional labeling}

Overall, users were surprisingly consistent in assigning emotional labels to pieces of music, with half of the users agreeing on at least one emotional category in $76 \%$ of the rated songs. A list of how many songs were placed in each category is presented in Table 2 The first column lists the number of songs assigned to each emotional category, where at least $50 \%$ of the study participants agreed on it as its most dominant emotional category. If one requires that at least $70 \%$ of the test users agree on an emotion, $35 \%$ (70) of the songs can be labeled, as listed in column 2. It seems that in such a (relatively small) group, there always will be some disagreement on the emotions connected with a song but that the majority of users can agree on at least one emotion for a high number of songs.

If one were to look at the emotion categories that received more than $50 \%$ of the votes (but did not require the emotion to be the highest rated of all emotions), 153 songs would be labeled, $63 \%$ (97 songs) of which in one category, $33 \%$ (50 songs) in two categories and 4\% (6 songs) in three categories.

In this case, the most highly correlated classes are: sadness/serenity, which appear in 29\% (16 times); hostility/self-assurance, which appear in 23\% (13 times); and joviality/self-assurance, which appear in 16\% (9 times) of 56 songs in two or more classes together.

\subsection{SOM for all users}

To visualize the results of the study, a SOM [6] was trained on the Rhythm Pattern features [11] of the whole music collection (1000 songs). As only 200 of the songs were rated in this study, the rest were tagged as "unknown". For the "All Users" evaluation, the music pieces were put into one or more of the 


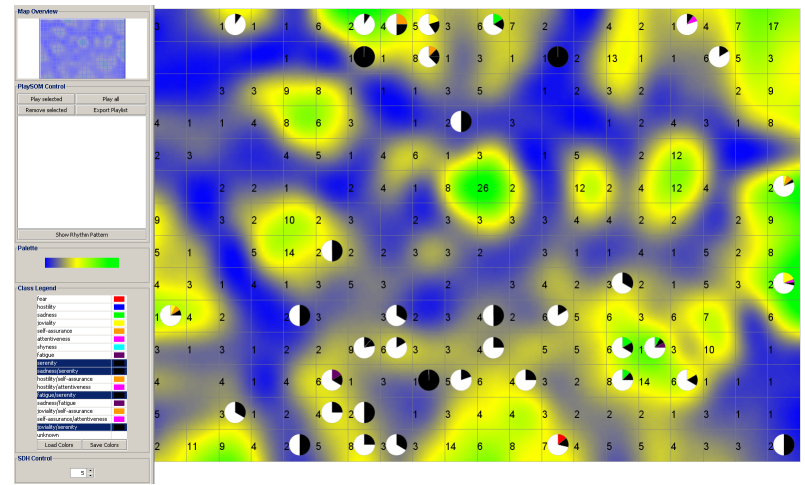

Fig. 1. Screen-shot of the SOM Viewer Software displaying serenity for all users.

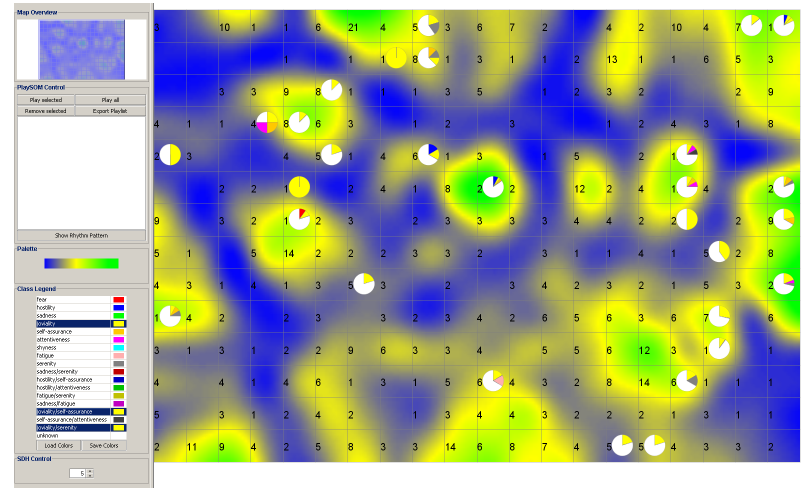

Fig. 2. Screen-shot of the SOM Viewer Software displaying joviality for all users.

emotion categories: A song was assigned the emotions most voted for if the emotions had at least $50 \%$ of the votes (that is 5 or more users agreed on the respective emotion). That is, if Song X was rated as "sad / lonely" by 5 users, as "scared / nervous" by 7 users, and as "timid / shy" by 7 users, it was assigned the categories "fear" and "shyness". Of the labeled songs, 92\% (140 songs) were assigned one category, and 8\% (12 songs) were assigned two categories.

Of the 200 rated songs, those that were not put into at least one and at most two classes were also tagged as "unknown" to keep the visualizations concise and readable. In total, 152 songs were labeled with one or two emotions and 848 as "unknown". This shows that at least $50 \%$ of the users agreed on at least one emotion category for more than $76 \%$ of the rated songs.

The SOM was then visualized with the SOMLib Viewer software, displaying for each emotion class which SOM units had a song of that class mapped onto it. Figure 1 provides an example visualization for the emotion class "serenity" for all users. It displays the SDH Visualization [9], revealing the sound similarity cluster structure as Islands of Music. On top of these the black pie charts show the fraction of songs on the respective unit with the class label serenity, 
plus, obviously, the fraction of remaining unlabeled songs in the complete data collection on those units. Quite a large number of songs (45) were assigned to this emotional category. They end up sub-divided into a big cluster on the lower border and a smaller cluster on the upper central border of the visualization.

Figure 2 provides the same visualization for the "joviality" class. These songs concentrate in two distinct areas, namely the upper left quarter and the righthand border, indicating two loose clusters. For the remaining emotions, the locations can be briefly summarized as follows:

Fear: There are very few (4) songs marked with this label. This does not warrant any conclusion on the distribution of "fear".

Hostility: Songs that are put in the category "hostility" seem to concentrate in the upper right region of the visualization and form small clusters.

Guilt: No songs were put into the "guilt" category by five or more users, so there are no songs for "guilt" on the visualization.

Sadness: Songs in this category seem to concentrate in the lower right area, and a tight cluster stands out in the middle of the lower right quarter.

Self-Assurance: Songs in the category "self-assurance" are rather scattered, but their concentration is much higher in the upper half of the visualization. One can imagine loose clusters there.

Attentiveness: Songs in the category "attentiveness" almost exclusively appear on the right-hand border.

Shyness: There were only two songs marked with this label. This does not warrant any conclusion on the distribution of "shyness".

Fatigue: Songs in this category are few, there seems to be a cluster in the lower left quarter and a few scattered ones in the right half.

Surprise: No songs were put into the "surprise" category by five or more users, so there are no songs for "surprise" on the visualization.

Figure 3 contains a (manually produced) overview on where one can detect clusters of emotions. It is just a rough sketch, rather than a density estimation, but it nonetheless permits some conclusions: The emotions cannot be separated completely, but they overlap - as is to be expected if one takes into account that feelings mix and music carries a lot of emotional information. However, it seems that the calmer and quieter emotions come to lie in the lower half of the visualization and the more aroused feelings can be found in the upper half - with the exception of the "serenity" cluster in the upper half. On the right-hand edge there seems to be an area of strong and cheerful music - the overlapping clusters of the right "joviality" cluster and the attentiveness area. The lower "serenity" cluster encompasses "fatigue" and "sadness", and "self-assurance" overlaps with or encloses "joviality", "serenity", "hostility", and "attentiveness".

Thus it may indeed be possible to generally derive the emotion connoted with a piece of music, though the classes, the classification procedure, and the data collection probably could be improved. 


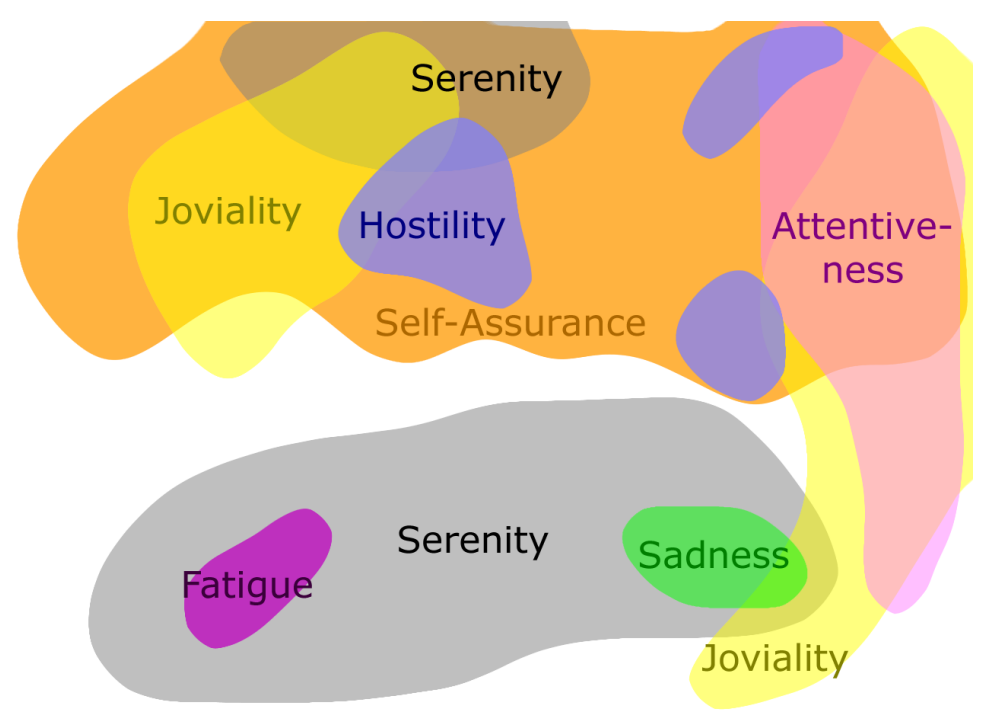

Fig. 3. Rough map of the distribution of the emotions on the SOM SDH visualization.

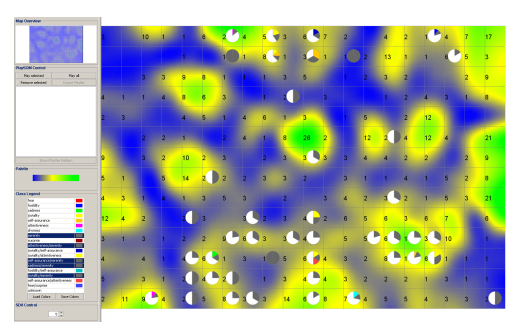

(a)

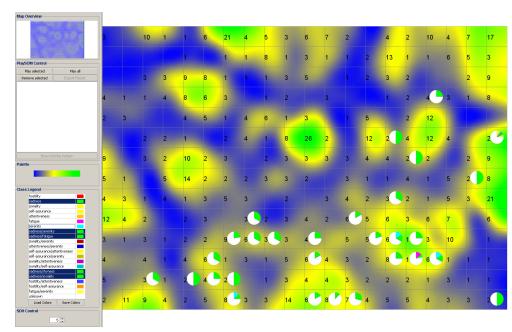

(b)

Fig. 4. Screen-shot of the SOM Viewer Software displaying serenity for (a) User A and (b) User B.

\subsection{SOM for individual users}

This section describes an interesting point found by comparing visualizations of individual user ratings. It shows how much the appraisal of music can differ between different people. Again, only songs in one or two emotion categories were used, thus, for the ten user results, between 195 and 78 songs have been assigned an emotion class in the visualizations.

Emotional appraisal can differ through individual mood or character. Comparing two users, called "User A" and "User B" here, User B has classified a lot more songs as "sad / lonely" than User A: See Figure 4(b) for User B's distribution of 36 sad songs. User A on the other hand has only rated 3 songs as "sad / lonely", which come to lie on the bottom border of the respective visualization. 
In User B's visualization, there seem to be one or two very tight "sadness" clusters in the lower half. These clusters cannot be found in User A's "sadness" visualization, however, very similar clusters can be found in the bottom half of the "serenity" visualization, marked on Figure 4(a) It further shows, that the two users disagree on the labels they assign to music from the same cluster, with User B calling that music "sad/lonely", whereas User A attributes virtually the same music to the serenity cluster ("calm/relaxed"). From this, one may get the impression that User A is a "happier" person or was in a more positive mood when taking the test. This is supported by the fact that User A rated more than twice as many songs (86) as "happy / cheerful" as User B (with 33 songs).

The emotional categories "sadness" and "serenity" are also the most highly correlated ones, hinting at them being related to each other. In the global overview map of all emotions for all users, the sadness cluster in the bottom right area constitutes a sub-cluster of the larger serenity cluster on the bottom half of the map.

\section{Conclusions}

We analyzed the potential of using emotional labels as descriptors for mapbased access to music libraries. Emotional categories from the PANAS-X Manual were used to label pieces of music, yielding a high consistency in labels assigned by different users. Furthermore, in a parallel process, the music was clustered according to psycho-acoustic features extracted from the audio files using a SOM. It showed a high consistency with respect to the labels assigned, i.e. clusters of different emotions appear on the SOM visualization, showing sensible correlation between emotions for music in the various regions. Emotions perceived as related are also located in neighboring map regions. Thus, emotions may be derivable in an automatic way by training classifiers. We are currently investigating in how far this automatic classification can achieve acceptable performance using a set of state of the art machine learning algorithms.

Still, the emotion categories from the PANAS-X Manual need to be rethought and adapted according to the needs of the music listeners. For example, it might not be necessary to include categories such as "guilt" or "surprise" if the listeners do not use them or if different users do not agree on what is to be placed in those classes.

Different users associate different feelings with a given piece of music, but for quite a lot of songs the majority of users agree on the connoted emotions. Also, not all emotions can automatically and reliably be derived from the features used, but for some emotions the features give a good idea of which emotional categories the song could be placed in.

Finally, with something so subjective as emotion, it might be more promising to build individual emotion classifiers for each listener than to try and derive a general notion of what song belongs to which emotional class. 


\section{References}

1. Sally Jo Cunningham, J. Stephen Downie, and David Bainbridge. "the pain, the pain": Modelling music information behavior and the songs we hate. In Proc. 6th Intl. Conf. on Music Information Retrieval (ISMIR 2005), pages 474-477, London, UK, September 11-15 2005.

2. M. Dittenbach, R. Neumayer, and A. Rauber. Playsom: An alternative approach to track selection and playlist generation in large music collections. In Proc. 1st Intl. Workshop on Audio-Visual Content and Information Visualization in Digital Libraries (AVIVDiLib 2005), pages 226-235, Cortona, Italy, May 4-6 2005.

3. Paul R. Farnsworth. The social psychology of music. Dryden Press, 1958.

4. Alf Gabrielsson and Patrik N. Juslin. Emotional expression in music. In Richard J. Davidson, Klaus R. Scherer, and H. Hill Goldsmith, editors, Handbook of Affective Sciences, pages 503-534. Oxford University Press, 2002.

5. Kate Hevner. Experimental studies of the elements of expression in music. In American Journal of Psychology, volume 48, pages 246-268, 1936.

6. T. Kohonen. Self-Organizing Maps, volume 30 of Springer Series in Information Sciences. Springer, Berlin, 3rd edition, 2001.

7. Kang-Kue Lee and Kyu-Sik Park. Robust feature extraction for automatic classification of Korean traditional music in digital library. In Proc. 8th Intl Conf. on Asian Digital Libraries (ICADL 2005), pages 167 - 170, Bangkok, Thailand, December 12-15 2005. Springer.

8. Tao Li and Mitsunori Ogihara. Detecting emotion in music. In Proceedings of the 4 th Intl. Conf. on Music Information Retrieval (ISMIR 2003), Baltimore, Maryland (USA), October 26-30 2003.

9. E. Pampalk, A. Rauber, and D. Merkl. Content-based organization and visualization of music archives. In Proc. of ACM Multimedia 2002, pages 570-579, Juan-les-Pins, France, December 1-6 2002. ACM.

10. A. Rauber and M. Frühwirth. Automatically analyzing and organizing music archives. In Proc. 5th Europ. Conf. on Research and Advanced Technology for Digital Libraries (ECDL 2001), Darmstadt, Germany, Sept. 4-8 2001. Springer.

11. A. Rauber, E. Pampalk, and D. Merkl. The SOM-enhanced JukeBox: Organization and visualization of music collections based on perceptual models. Journal of New Music Research, 32(2):193-210, June 2003.

12. Andreas Rauber. Creation and exploration of musical information spaces. In Proc. Intl Conf on Digital Libraries (ICDL2004),pages 741-748. TERI, 2004.

13. James A. Russell. Core affect and the psychological construction of emotion. Psychological Review, 110(1):145-172, January 2003.

14. Auke Tellegen, David Watson, and Lee Anna Clark. On the dimensional and hierarchical structure of affect. Psychological Science, 10(4):297-303, July 1999.

15. Robert E. Thayer. The Biopsychology of Mood and Arousal. Oxford University Press, 1989

16. G. Tzanetakis, G. Essl, and P.R. Cook. Automatic musical genre classification of audio signals. In Proc. Intl Symp. on Music Information Retrieval (ISMIR), pages 205-210, Bloomington, Indiana, October 15-17 2001.

17. David Watson and Lee Anna Clark. The PANAS-X Manual for the Positive and Negative Affect Schedule - Expanded Form. The University of Iowa, 1994. http: //www.psychology . uiowa.edu/faculty/Clark/PANAS-X.pdf

18. Dan Yang and WonSook Lee. Disambiguating music emotion using software agents. In Proc. 5th Intl Conf. on Music Information Retrieval (ISMIR 2004), Barcelona, Spain, October 10-14 2004. 